\title{
Vibrational dynamics of solid molecular nitrogen to megabar pressures
}

\author{
A. F. Goncharov, E. Gregoryanz, H.-K. Mao, and R. J. Hemley \\ Geophysical Laboratory and Center for High Pressure Research, Carnegie Institution of Washington \\ 5251 Broad Branch Road, N. W., Washington, D. C. 20015 \\ E-mail: goncharov@gl.ciw.edu \\ Received July 2, 2001
}

\begin{abstract}
We report the results of Raman and synchrotron infrared absorption measurements of several molecular phases of solid nitrogen to pressures above $100 \mathrm{GPa}(300 \mathrm{~K})$. Low-temperature vibrational spectra to $45 \mathrm{GPa}$ are also presented. The changes in Raman and infrared spectra at $60 \mathrm{GPa}$ and $300 \mathrm{~K}$ are interpreted as arising from the $\varepsilon \rightarrow \zeta$ transition reported at low temperature. The character of splitting of the Raman vibron $v_{2}$ observed at $25 \mathrm{GPa}$ and low temperatures differs from that previously reported, a difference that we ascribe to different experimental procedures employed and metastability of the low-temperature phase.
\end{abstract}

PACS: $62.50 .+p, 78.30 .-\mathbf{j}$

\section{Introduction}

With the recent progress in diamond-anvil cell techniques (see Ref. 1), a growing number of examples of orientational ordering and subsequent transformations to framework structures in pressurized molecular solids have been documented [2-6]. For transformation to framework structure, information about the type and character of the ordering is important, since it affects the energy barriers associated with the transition. Solid nitrogen is an important system for such studies because of stability and simple electronic structure of the isolated molecule. Moreover, solid nitrogen has been well studied theoretically, and accurate experimental data provide an important test of condensed matter theory [7-12].

Solid nitrogen has a complex phase diagram with a wealth of molecular phases that differ in the types of orientational ordering and crystal structures formed [5,13-33]. The ordering of the low pressuretemperature $\alpha$ and $\gamma$ phases is controlled by quadrupole-quadrupole interactions, whereas at higher pressures a class of molecular structures $\left(\delta, \delta_{\text {loc }}, \varepsilon\right.$, $\zeta)$ stabilized by additional anisotropic intermolecular interactions is found [34]. The $\delta$ phase is proposed to have a disordered cubic structure (space group $P m 3 n$ ) [20] with two different types of molecules exhibiting sphere- and disk-like disorder and giving rise to two classes of vibron bands $v_{1}$ and $\mathrm{v}_{2}$. With increasing pressure and/or decreasing temperature, nitrogen molecules exhibit orientational order through a sequence of the phase transformations (to $\delta_{\text {loc }}$ and then $\varepsilon$ ) as determined by vibrational spectroscopy and $\mathrm{x}$-ray diffraction [27-31]. The structure of the $\varepsilon$ phase is rhombohedral (space group $R \overline{3} c$ ) which can be viewed as a distortion of cubic [23].

Theoretical calculations have been contradictory in their predictions of stable molecular ordered phases [10-12]. In particular, Monte Carlo (MC) [10] and molecular dynamics (MD) [11] simulations based on proposed intermolecular potential favor tetragonal structures, while $a b$ initio calculations [12] predicted the stability of $R \overline{3} c$ phase in agreement with experiment. Recent MC calculations $[35,36]$ pointed out the importance of using accurate atom-atom potentials (especially anisotropic terms) to obtain adequate results.

At $20-25 \mathrm{GPa}$ and $10 \mathrm{~K}$ a transformation has been found by Raman spectroscopy [21] and confirmed by later Raman and IR measurements [33]. No x-ray data are currently available for this phase (called $\zeta$ ). According to vibrational spectroscopy data, its structure has a strong similarity to phases $\varepsilon$ and $\delta$. It has been inferred to have $R 3 c$ symmetry [21], but more recent observations do not support this interpretation [33]. The $\varepsilon-\zeta$ phase boundary has been extended recently to $180 \mathrm{~K}$ and $40 \mathrm{GPa}$ [33]. At low temperatures, a significant 
region of metastability is reported [33], making difficult to clarify the mechanism of the transformation.

At room temperature, the vibrational spectroscopy data are still contradictory concerning a number and nature of the transformations above $20 \mathrm{GPa}$. A sequence of new phases has been reported on the basis of several branchings of the Raman vibron modes [22], including one just above $20 \mathrm{GPa}$ [27]. In contrast, $\mathrm{x}$-ray studies indicate the stability of $\varepsilon$ phase to $50 \mathrm{GPa}[26,30]$. A change of $\mathrm{x}$-ray diffraction pattern was observed above $60 \mathrm{GPa}$ [25], but interpretation requires additional measurements. Most recent Raman and IR measurements to $42 \mathrm{GPa}$ show clear correspondence between the number of observed lattice and vibron modes and group-theoretical predictions for the $\varepsilon$ phase [33]. Here we report the results of a combined Raman and synchrotron IR high-pressure study of $\mathrm{N}_{2}$ to $40 \mathrm{GPa}$ at low temperatures and beyond $100 \mathrm{GPa}$ at room temperature.

\section{Experimental}

Samples of nitrogen were loaded in diamond anvil cells at $0.2 \mathrm{GPa}$ using a standard gas-loading technique. The low-temperature experiment was performed at the NSLS (beamline U2A). The description of our experimental setup has been published elsewhere [37]. Typical spectral resolution was about $4 \mathrm{~cm}^{-1}$ in both IR and Raman measurements. A diamond cell with the sample at $8 \mathrm{GPa}$ was cooled to $15 \mathrm{~K}$ in a continuous-flow He cryostat. The cryostat is equipped with windows that are changeable at low temperature, which allows in situ IR and Raman/ruby fluorescence measurements without changing $P-T$ conditions on the sample [38]. Pressure can be controlled from the outside by a mechanical change in load on the diamond cell lever-arm through vacuum feedthroughs. In the room-temperature experiments we used diamond-anvil cells with external heating capabilities. This allows us to release internal stresses that build up during a loading of the cell by heating the sample to $200-400{ }^{\circ} \mathrm{C}$.

\section{Results}

The sequence of Raman spectra measured as a function of pressure at low temperatures is shown in Fig. 1. At 17.5 GPa we observe two Raman peaks in the vibron region - slightly broadened $v_{2}$ (lower frequency) and $v_{1}$. This is in excellent accord with the experiments for the $\varepsilon$ phase (see also Refs. 21 and 33). Low-frequency lattice modes (not shown)

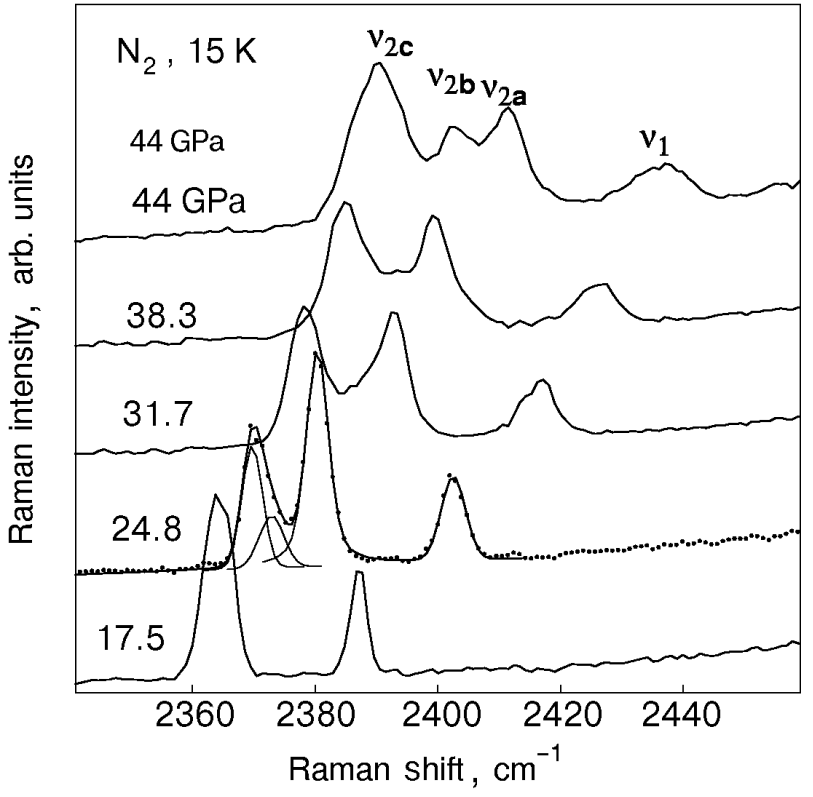

Fig. 1. Raman spectra of nitrogen through the low-temperature transition to the $\zeta$ phase. The spectra are shifted in vertically for clarity. The spectrum at $24.8 \mathrm{GPa}$ (points) is shown along with the fitting to multiple components (Voigt profiles).

are also in agreement with previous studies for $\varepsilon-\mathrm{N}_{2}[21,33]$. Increasing pressure splits the $v_{2}$ peak, so three components can be seen. This splitting becomes obvious at the highest pressure ( $44 \mathrm{GPa})$, while at 24-38 $\mathrm{GPa}$ peak fitting is required to reveal the two components (e.g., the spectrum at $24.8 \mathrm{GPa}$ in Fig. 1). Only a slight broadening of the $v_{1}$ peak is observed as the pressure is increased. Lattice modes also change dramatically at $18^{-}$

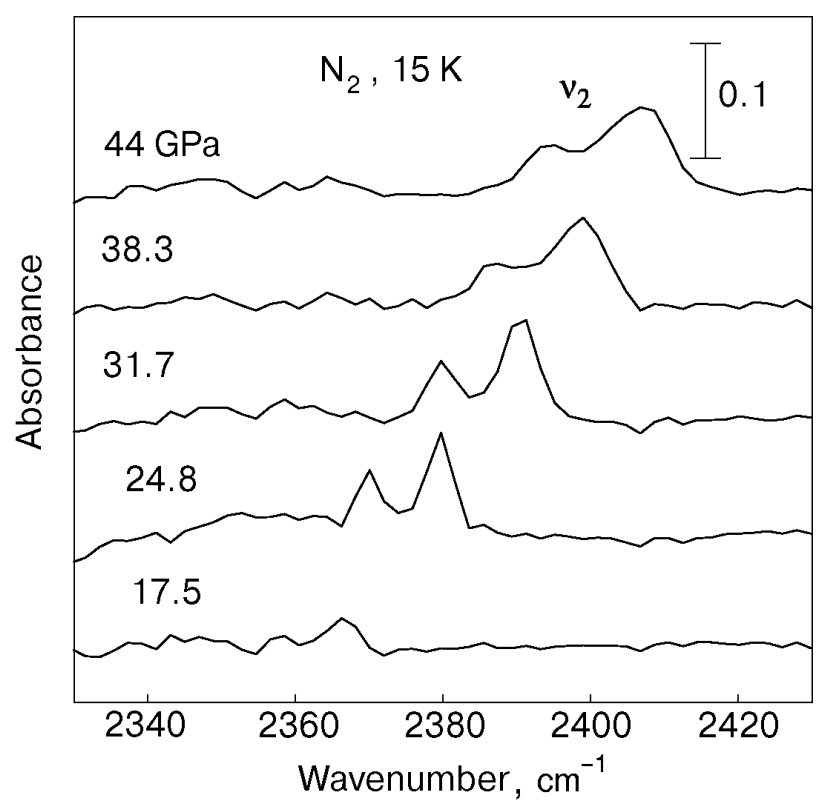

Fig. 2. Infrared absorption spectra of nitrogen at $15 \mathrm{~K}$ as a function of pressure. The spectra are shifted in vertically for clarity. 
$25 \mathrm{GPa}$, which is beyond the scope of the present paper.

Infrared spectra at $17.5 \mathrm{GPa}$ (Fig. 2) show a very weak absorption in a spectral range of the $v_{2}$ vibron. The absorbance increases substantially at 18-25 GPa, so a doublet of IR vibrons is clearly visible at higher pressure. As for the Raman bands, a moderate broadening of the IR peaks is observed as the pressure is increased.

The pressure dependence of the observed Raman frequencies is shown in Fig. 3. Corresponding data from Ref. 33 are also shown for comparison. Earlier results [21] for the $v_{2}$ multiplet are very close to those reported in Ref. 33 and are not shown for clarity. For the $v_{1}$ band, our low-temperature data are very close to those measured at the room temperature (see also Ref. 32). The substantial difference in the pressure dependence of the frequency of the Raman $v_{2}$ multiplet will be discussed later. The pressure dependences of the Raman and infrared frequencies are compared in the inset of Fig. 3. We find that Raman and infrared frequencies do not coincide (cf. Ref. 33). Unlike the Raman data, our infrared frequencies are in good agreement with those reported in Ref. 33 in the pressure range overlapped by the two studies (to $25.2 \mathrm{GPa}$ ).

Raman spectra measured close to room temperature are presented in Fig. 4. At $60 \mathrm{GPa}$ Raman spectra already contain more vibron bands (four)

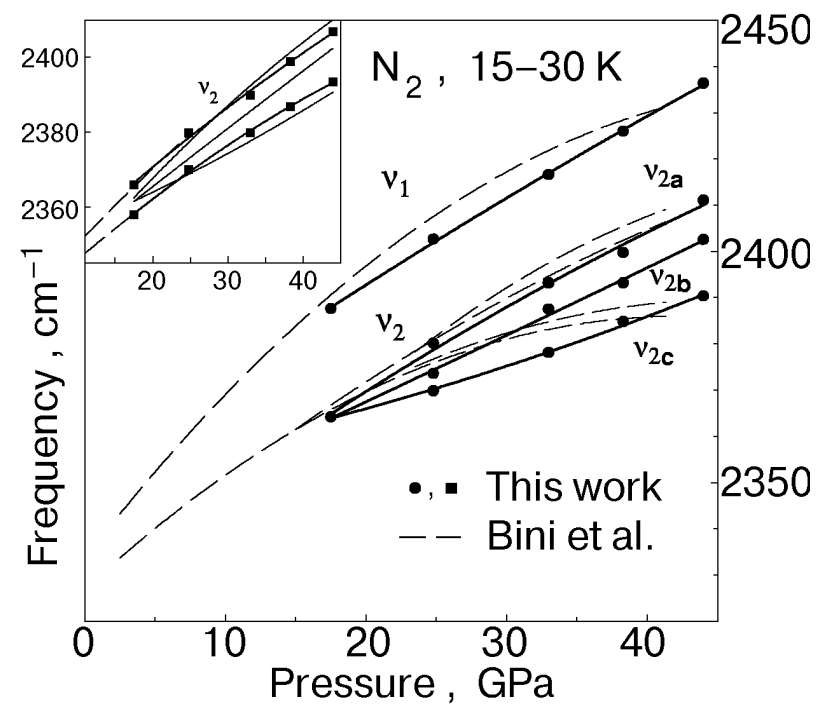

Fig. 3. Raman vibrational frequencies as a function of pressure at $15 \mathrm{~K}$. Filled circles are the Raman data from this work. The solid thick solid lines are the guides to the eye. The dashed lines are the Raman data from Ref. 33. The inset shows the comparison between Raman and infrared frequencies. Filled squares are the infrared data from this work. The solid thick solid lines are the guides to the eye. Thin solid lines are the Raman data from this work. The dashed lines are the infrared data from Ref. 33. that is allowed by group theory for the $R \overline{3} c$ symmetry of $\varepsilon-\mathrm{N}_{2}$ (three) [21]. Surprisingly, at this pressure we did not observe the low-frequency shoulder of the most intense $v_{2 c}$ Raman band, as reported in Ref. 32. Increasing of pressure through the $60 \mathrm{GPa}$ range gives rise to a new Raman peak $\left(v_{2 b}\right)$, designated by arrow in Fig. 4 (see also Ref. 32). A similar observation was made when cooling down from approximately the same starting point (inset in Fig. 4). At this pressure (and room temperature) we also observed an increase in intensity of the infrared vibron [3]. Raman spectra in the lattice mode range show splittings of the characteristic bands of the low-pressure phase and appearance of new low-frequency bands. The splitting of the lattice modes increases with pressure with multiplets evolving into distinct bands. Representative Raman spectra in the lattice mode region are presented in Fig. 5. The complexity of the spectra suggests a large number of molecules and low symmetry of the unit cell. The spectra at $95 \mathrm{GPa}$ agree well with those presented in Ref. 32 in terms of the number and positions of bands, with the exception of a very weak additional vibron band near $2473 \mathrm{~cm}^{-1}$. With further pressure increase, the intensity of the lower frequency vibron increases and all other vibron peaks gradually vanish [3,22]. Similarly, in the lattice-mode region the intensity of the $500 \mathrm{~cm}^{-1}$ band increases prior to the transition to the non-

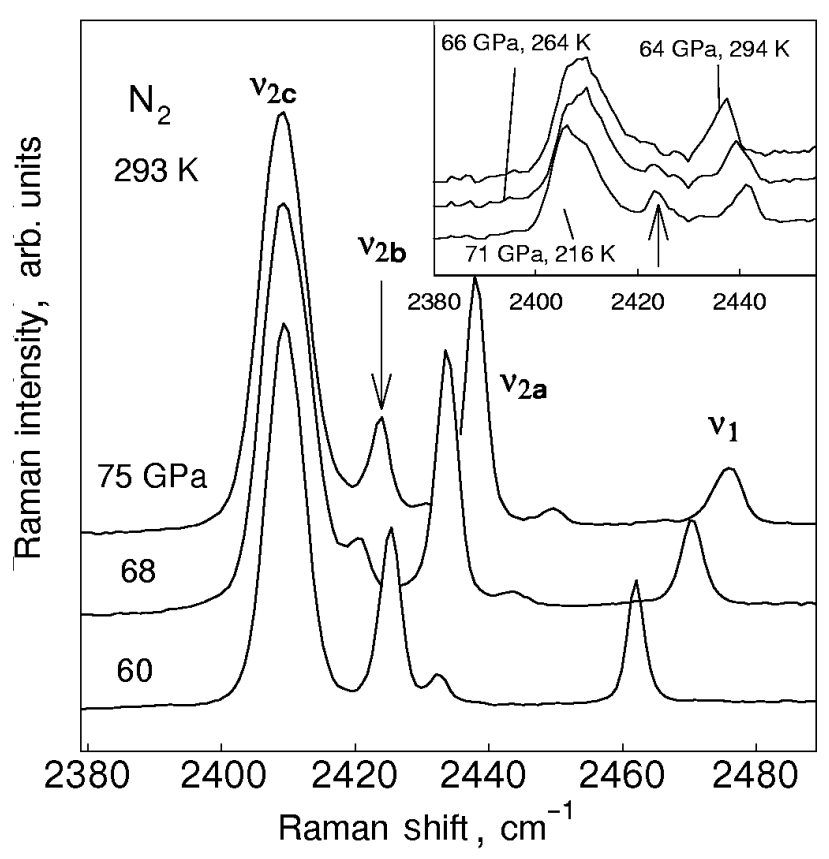

Fig. 4. Raman spectra of the nitrogen vibrons through the transition to the $\zeta$ phase at room temperature. The inset shows the variation of spectra with temperature. The arrow designates a new peak, that appears at the transition. The spectra are shifted in vertically for clarity. 


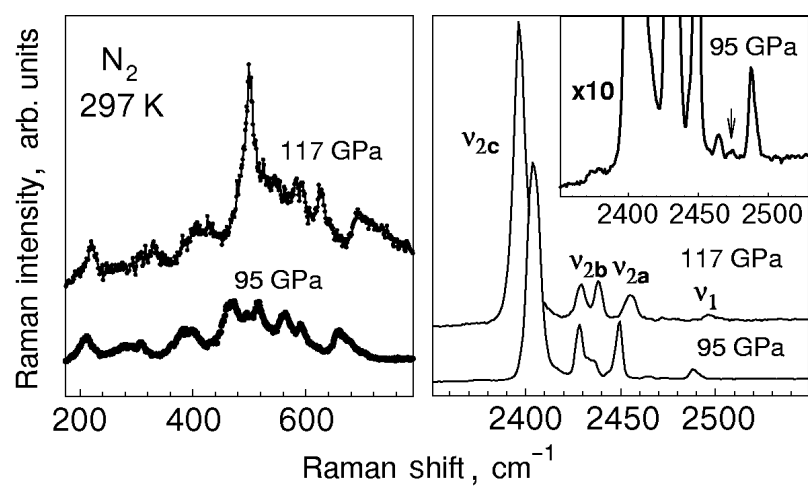

Fig. 5. Raman spectra of nitrogen at 95 and $117 \mathrm{GPa}$. The inset shows a weak vibron peaks (designated by arrow) that develops under high pressure. The spectra are shifted in vertically for clarity.

molecular phase. This behavior is will be examined in detail elsewhere.

\section{Discussion}

The splitting of the vibron bands and change in the lattice mode spectrum observed at low temperature indicate a phase transformation related to orientational ordering of the nitrogen molecules. The phase diagram of Ref. 33 suggests that these changes correspond to the $\varepsilon \rightarrow \zeta$ transition. Qualitatively, our data and those presented in Refs. 21 and 33 show similar trends, but detailed comparison shows different Raman spectra for the highpressure phase (Fig. 3). We believe that the disagreement arises from the use of different experimental procedures and the nature of the highpressure phase (or phases). In contrast to experiments reported in Refs. 21 and 33, we changed pressure at low temperature. It is useful to note that when infrared spectra were measured in a manner similar to ours [33], the results from both studies agree very well (inset to Fig. 3). The evidence that the properties of the high-pressure phase depend on the thermodynamic path, suggests that this phase is not thermodynamically stable (i.e., metastable at the indicated $P-T$ conditions). This is supported by observations of a large hysteresis of the transition at low temperatures [33]. An alternative (but related) explanation is that different properties of the highpressure phase arise from relatively large pressure inhomogeneities in our experiment (since we changed pressure at low temperature). This is indicated by broadening of Raman and infrared bands at higher pressures (Figs. 1 and 2).
Changes in Raman and infrared spectra above $60 \mathrm{GPa}$ at room temperature are very similar to those observed at low temperatures. Moreover, the reported transition boundary [33] extrapolated to high pressure and temperature matches this roomtemperature point. According to the observed Raman and infrared spectra, the vibrational properties of the high-pressure phase are very similar at room and low temperature. Thus, we will consider it to be the same phase $(\zeta)$.

In view of the absence of sufficient $\mathrm{x}$-ray data for the $\zeta$ phase, we can only speculate on its crystal structure. The number of the vibron modes (in either our experiment or those reported in Ref. 32) exceeds that predicted for $R 3 \mathrm{c}$ structure based on the space group theory proposed in Ref. 21. According to Ref. 32, the increase of number of vibron modes is due to the increase in the number of different site symmetries occupied by $\mathrm{N}_{2}$ molecules. Following this idea, up to 5 different site symmetry positions should be invoked to explain the observed number of Raman vibron peaks above $60 \mathrm{GPa}$, which does not seems plausible. A critical examination of the spectra of Ref. 32 shows that this number can probably be reduced to 3 according to the number of observed distinct peaks in the Raman exitations of the guest molecules. Thus, it seems natural to propose that the branching of vibron modes is related to sequential lifting of degeneracy of the $v_{2}$ term of the cubic $\delta$ phase. In the first stage ( $\delta$ to $\varepsilon$ transition), the $v_{2}$ band splits into $A_{1 g}$ and $E_{g}$ components by the crystal field. In the second one ( $\varepsilon$ to $\zeta$ transition), the symmetry is further reduced (to orthorhombic or monoclinic), with doubly degenerate level splitting into two singlets. Additional splitting (vibrational or Davydov-type) of these major components could be caused by intermolecular interactions. This is related to a possible increase in the number of molecules in the unit cell as well as associated symmetry lowering. High-quality diffraction data are required to examine these hypotheses.

\section{Conclusions}

We find that the properties of the high-pressure, low-temperature phase of nitrogen obtained by «cold» compression are different from those for the phase quenched from high temperature. This suggests that the $\zeta$ phase is metastable and/or transitions to it are sensitive to nonhydrostatic effects. Raman and infrared spectra of $\varepsilon-\mathrm{N}_{2}$ above $40 \mathrm{GPa}$ and $\zeta-\mathrm{N}_{2}$ are not compatible with $R \overline{3} c$ and $R 3 c$ symmetries proposed in Ref. 21 because the number of vibron bands is larger than predicted for the 
standard structures based on these space groups. This increase in the number of bands is probably related to the additional lowering of the symmetry and multiplication of the size of the unit cell.

The present vibrational spectroscopy data provide additional constraints on the structure and properties of the high-pressure phases. They also suggest that known phases are not necessarily thermodynamically stable in the $P-T$ region in which they can be observed. As for other molecular crystals, sluggish kinetics can complicate the determination of the true thermodynamic phase diagram (see, e.g., Ref. 39). Further theoretical and experimental effort is necessary to obtain a better understanding of the phase diagram of nitrogen at these high-pressure conditions.

This work was supported by the NSF. The NSLS is supported by DOE.

1. R. J. Hemley and H.-K. Mao, in: Encyclopedia of Applied Physics, G. L. Trigg (ed.), New York, VCH Publishers, vol. 18 (1997), p. 555.

2. V. Iota, C. S. Yoo, and H. Cynn, Science 283, 1510 (1999).

3. A. F. Goncharov, E. Gregoryanz, H.-K. Mao, Z. Liu, and R. J. Hemley, Phys. Rev. Lett. 85, 1262 (2000).

4. M. I. Eremets, R. J. Hemley, H. K. Mao, and E. Gregoryanz, Nature 411, 170 (2001)

5. E. Gregoryanz, A. F. Goncharov, R. J. Hemley, and H.-K. Mao, Phys. Rev. B64, 52103 (2001).

6. E. Katoh, H. Yamawaki, H. Fujihisa, M. Sakashita, and K. Aoki, Phys. Rev. B61, 119 (2000).

7. A. K. McMahan and R. LeSar, Phys. Rev. Lett. 54, 1929 (1985).

8. R. M. Martin and R. J. Needs, Phys. Rev. B34, 5082 (1986).

9. C. Mailhiot, L. H. Yang, and A. K. McMahan, Phys. Rev. B46, 14419 (1992).

10. J. Belak, R. LeSar, and R. D. Etters, J. Chem. Phys. 92, $5430(1990)$

11. S. Nosé and M. L. Klein, Phys. Rev. Lett. 50, 1207 (1983).

12. R. D. Etters, V. Chandrasekharan, E. Uzan, and K. Kobashi, Phys. Rev. B33, 8615 (1986).

13. C. A. Swenson, J. Chem. Phys. 23, 1963 (1955).

14. R. L. Mills and A. F. Schuch, Phys. Rev. Lett. 23, 1154 (1969).

15. F. Schuch and R. L. Mills, J. Chem. Phys. 52, 6000 (1970).
16. J. R. Brookeman and T. A. Scott, J. Low. Temp. Phys. 12 , 491 (1973).

17. W. E. Streib, T. H. Jordan, and W. N. Lipscomb, J. Chem. Phys. 37, 2962 (1962).

18. R. LeSar, S. A. Ekberg, L. H. Jones, R. L. Mills, L. A. Schwalbe, and D. Schiferl, Solid State Commun. 32, 131 (1979).

19. S. Buchsbaum, R. L. Mills, and D. Schiferl, J. Phys. Chem. 88, 2522 (1984).

20. D. T. Cromer, R. L. Mills, D. Schiferl, and L. A. Schwalbe, Acta Crystallogr. B37, 8 (1981).

21. D. Schiferl, S. Buchsbaum, and R. L. Mills, J. Phys. Chem. 89, 2324 (1985).

22. R. Reichlin, D. Schiferl, S. Martin, C. Vanderborgh, and R. L. Mills, Phys. Rev. Lett. 55, 1464 (1985).

23. R. L. Mills, B. Olinger, and D. T. Cromer, J. Chem. Phys. 84, 2837 (1986).

24. S. Zinn, D. Schiferl, and M. F. Nicol, J. Chem. Phys. 87, 1267 (1987)

25. A. P. Jephcoat, R. J. Hemley, H.-K. Mao, and D. E. Cox, Bull. Am. Phys. Soc. 33, 522 (1988).

26. H. Olijnyk, J. Chem. Phys. 93, 8968 (1990).

27. H. Schneider, W. Haefner, A. Wokaun, and H. Olijnyk, $J$. Chem. Phys. 96, 8046 (1992).

28. M. I. M. Scheerboom and J. A. Schouten, Phys. Rev. Lett. 71, 2252 (1993).

29. M. I. M. Scheerboom and J. A. Schouten, J. Chem. Phys. 105, 2553 (1996).

30. M. Hanfland, M. Lorenzen, C. Wassilew-Reul, and F. Zontone, in: Abstracts of the International Conference on High Pressure Science and Technology, Kyoto, Japan, 1997, p. 130.

31. R. Bini, M. Jordan, L. Ulivi, and H. J. Jodl, J. Chem. Phys. 108, 6869 (1998).

32. H. Olijnyk and A. P. Jephcoat, Phys. Rev. Lett. 83, 332 (1999).

33. R. Bini, L. Ulivi, J. Kreutz, and H. J. Jodl, J. Chem. Phys. 112, 8522 (2000).

34. V. G. Manzhelii and Y. A. Freiman, Physics of Cryocrystals, American Institute of Physics, College Park MD (1997).

35. A. Mulder, J. P. J. Michels, and J. A. Schouten, J. Chem. Phys. 105, 3235 (1996).

36. A. Mulder, J. P. J. Michels, and J. A. Schouten, Phys. Rev. B57, 7571 (1998).

37. A. F. Goncharov, V. V. Struzhkin, R. J. Hemley, H.-K. Mao, and Z. Liu, in: Science and Technology of High Pressure, M. H. Manghnani, W. J. Nellis, and M. F. Nicol (eds.), Universities Press, Hyderabad, India, Honolulu, Hawaii (1999), vol. 1, p. 90.

38. Z. Liu, A. F. Goncharov, H. Mao, and R. Hemley, Eos Trans. AGU 81 (48), Fall Meet. Suppl., Abstract T71B-22 (2000)

39. R. Jeanloz, J. Geophys. Res. 92, 10352 (1987). 